\title{
Imposing essential boundary conditions in mesh-free methods ${ }^{\star}$
}

\author{
Sonia FERNÁNDEZ-MÉNDEZ and Antonio HUERTA \\ Laboratori de Càlcul Numèric (www-lacan.upc.es), Departament de Matemàtica \\ Aplicada III, E.T.S. de Ingenieros de Caminos, Canales y Puertos, Universitat \\ Politècnica de Catalunya, Jordi Girona 1, E-08034 Barcelona, SPAIN.
}

\begin{abstract}
Imposing essential boundary conditions is a key issue in mesh-free methods. The mesh-free interpolation does not verify the Kronecker delta property and, therefore, the imposition of prescribed values is not as straightforward as for the finite element method. The aim of this paper is to present a general overview on the existing techniques to enforce essential boundary conditions in Galerkin based mesh-free methods. Special attention is paid to the mesh-free coupling with finite elements for the imposition of prescribed values and to methods based on a modification of the Galerkin weak form. Particular examples are used to analyze and compare their performance in different situations.
\end{abstract}

Key words: essential boundary conditions, mesh-free methods, Lagrange multipliers, Nitsche's method, coupling with finite elements

\section{Introduction}

In spite of the important effort dedicated to mesh-free methods in the last decade, see [1-3] for a general presentation, there are still many aspects that require further research. For example, an efficient imposition of essential boundary conditions in Galerkin based mesh-free methods, such as the EFG method [4], the RKPM [5] or the CSPH method [6], is still an open issue.

\footnotetext{
ऋ Sponsored by Ministerio de Ciencia y Tecnología (grants: DPI2001-2204 and REN2001-0925-C03-01) and the Generalitat de Catalunya (grant: 2001SGR00257)

Email addresses: sonia.fernandez@upc.es (Sonia FERNÁNDEZ-MÉNDEZ), antonio.huerta@upc.es (Antonio HUERTA).
} 
In the mesh-free context, shape functions usually do not verify the Kronecker delta property. That is, the set of mesh-free shape functions is a partition of the unity, but the shape function associated to a particle does not vanish at other particles. Therefore, imposing Dirichlet boundary conditions is not as trivial as in the finite element method. In the recent years, many specific techniques for the implementation of essential boundary conditions in mesh-free methods have been developed.

These techniques can be classified in two main groups: (1) methods based on a modification of the weak form, such as the Lagrange multiplier method [4], the penalty method [7] and Nitsche's method [8,9], and (2) methods that can be interpreted as a modification of the interpolation shape functions, see for instance [10-12].

Methods in the first group consider a modified weak form and they allow the use of trial functions that do not vanish at the essential boundary. The Lagrange multiplier method is one of the most widely used because of its straightforward implementation in all kind of problems. This method introduces a new unknown function, the Lagrange multiplier. The interpolation
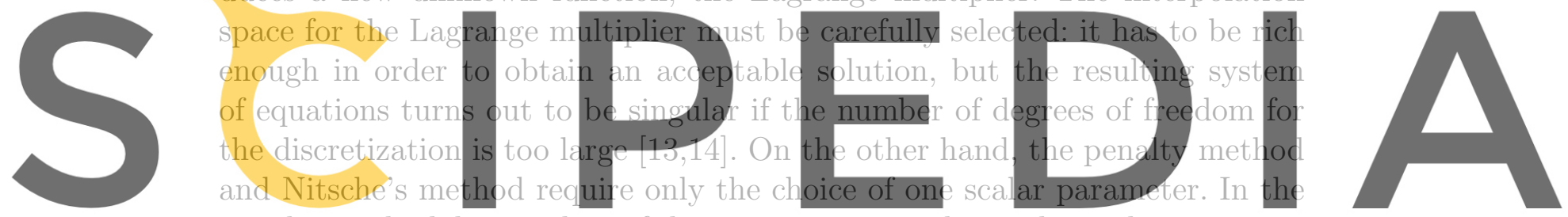

penalty method, large values of this parameter must be used in order to impose

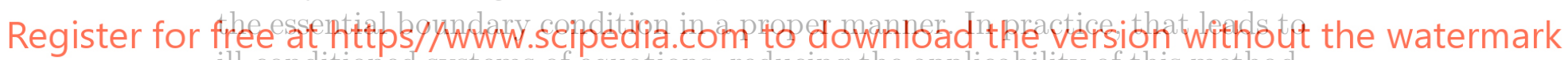

ill-conditioned systems of equations, reducing the applicability of this method.

On the contrary, Nitsche's method does not suffer of ill-conditioning. However,

the implementation of Nitsche's method is not as trivial as for the Lagrange multiplier method or the penalty method, in the sense that the modification of the weak form is different for each particular problem.

In the second group, several alternatives are also available. By introducing an extension of the dilation parameter at each particle, the mesh-free shape functions can be forced to verify the Kronecker delta property at the boundary [10]. A transformation method that expresses the mesh-free unknowns as a linear combination of nodal unknowns is proposed in [15]. This method allows the definition of shape functions that verify the delta property, thus, essential boundary conditions are easily enforced. D'Alembert principle is considered in [16] for mesh-free methods. It can be applied for the imposition of all kind of linear constraints. Orthogonality of the constraint matrix is assumed in order to express the unknowns as a linear combination of a set of generalized unknowns. With this generalized unknowns essential boundary conditions are directly imposed. Transformation methods are usually employed in transient problems, or evolution problems, and transformation matrices are computed 
only once.

Alternatively, the modification of the mesh-free shape functions to couple with a finite element interpolation near the essential boundary [11,12,17] allows to directly impose prescribed values. This implies the modification of the meshfree code in order to include finite elements. However, the modifications are only made at the interpolation level, and it can be easily applied to all kind of problems. Belytschko and coworkers [11] propose a coupled interpolation in the transition region, i.e. the area where both finite elements and particles have an influence. This coupled interpolation requires the substitution of finite element nodes by particles and the definition of ramp functions, thus the transition is of the size of one finite element and the interpolation is linear. A unified and general formulation for a continuous blending is presented in [12]. The continuous blending method allows the coupling of a mesh-free approximation with finite elements and, as commented in Section 2, it can be applied to the imposition of essential boundary conditions. It also allows enrichment of finite elements with particles. This approach has been generalized in [18] to get a nodal interpolation property. On the other hand, the bridging scale
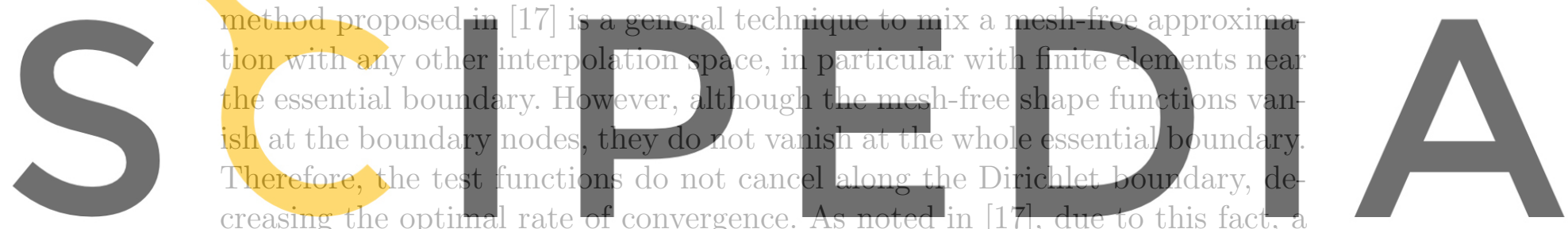

modified weak form must be used to impose the essential boundary condition

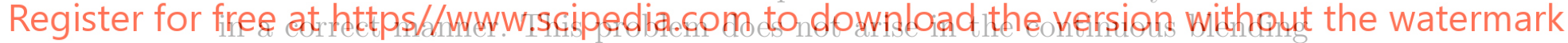

method [12]. A detailed comparison between the continuous blending method [12] and the bridging scale method [17], for the implementation of essential boundary conditions, can be found in [19].

The aim of this paper is to review and compare some of the most powerful techniques for the imposition of essential boundary conditions in mesh-free methods. Special attention is paid to the continuous blending of mesh-free methods with finite elements proposed in [12] and to methods based on a modification of the weak form. Although all the developments and examples are centered in the EFG method, all the results are also valid for other meshfree methods based on a Galerkin weak form, such as RKPM or CSPH. Section 2 recalls basic concepts on the continuous blending of mesh-free methods with finite elements and fully develops the approach for the imposition of prescribed values. Section 3 is devoted to review and compare three techniques based on a modification of the weak form: the Lagrange multiplier method and the penalty method, which are widely used in mesh-free methods, and Nitsche's method. Finally, in Section 4, two numerical examples corroborate the conclusions. 


\section{Coupling to finite elements: continuous blending method}

Given a set of particles $x_{i}$ in the domain $\Omega \subset \mathbb{R}^{n}$, mesh-less methods are based in a functional interpolation of the form

$$
u(x) \simeq u^{\rho}(x)=\sum_{i} N_{i}^{\rho}(x) u_{i}
$$

In the context of the EFG method [4], the mesh-free shape functions can be written as

$$
N_{i}^{\rho}(x)=\mathbf{P}\left(x_{i}\right)^{T} \boldsymbol{\alpha}(x) \phi\left(\frac{x-x_{i}}{\rho}\right)
$$

where $\mathbb{P}(x)=\left\{p_{0}(x), p_{2}(x), \ldots, p_{l}(x)\right\}^{T}$ includes a complete basis of polynomials of degree less or equal $m$ and the function $\phi(x)$ is the weighting function. It gives compact support to the shape function and this support is scaled by the dilation parameter $\rho$. The unknown vector $\boldsymbol{\alpha}(x)$ in $\mathbb{R}^{l+1}$ is determined imposing the so-called reproducibility or consistency condition. In fact, it is equivalent to a Moving Least Squares development, see [20]. This reproducibility condition imposes that the mesh-free approximation is exact
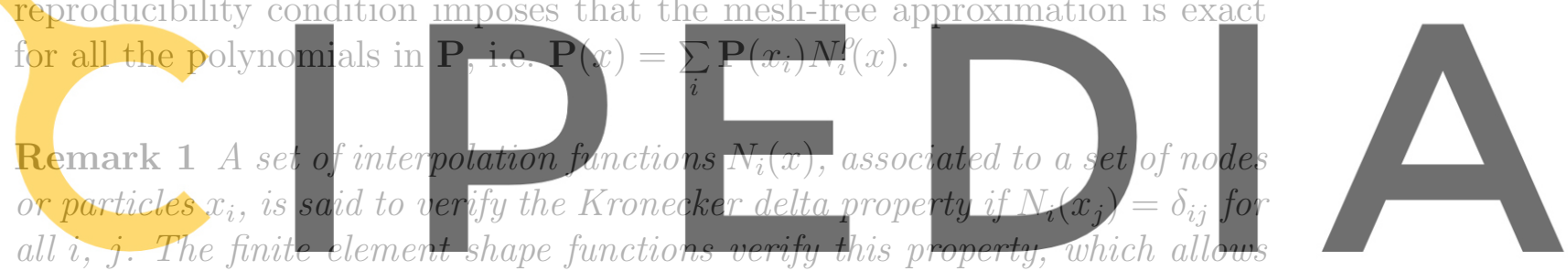

to directly enforce prescribed values (see Remark 3). However, as usual in

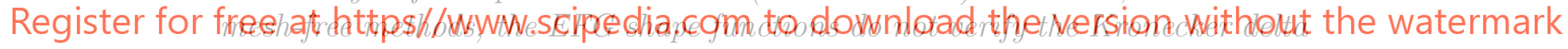
property. Therefore, $u^{\rho}\left(x_{i}\right) \neq u_{i}$ and imposing essential boundary conditions is not as trivial as for the finite element method.

Following this idea, Huerta and coworkers [12,21-23] propose a continuous blending of EFG and finite elements,

$$
u(x) \simeq \widetilde{u}(x)=\sum_{j \in \mathcal{J}} N_{j}^{h}(x) u_{j}+\sum_{i \in \mathcal{I}} \widetilde{N}_{i}^{\rho}(x) u_{i}=\pi^{h} u+\sum_{i \in \mathcal{I}} \widetilde{N}_{i}^{\rho}(x) u_{i}
$$

where the finite element shape functions $\left\{N_{j}^{h}\right\}_{j \in \mathcal{J}}$ are as usual, and the meshfree shape functions $\left\{\widetilde{N}_{i}^{\rho}\right\}_{i \in \mathcal{I}}$ take care of the consistency of the the approximation. $\pi^{h}$ denotes the projection operator onto the finite element space. That is, the span of some finite element shape functions characterized by an element mesh size $h$ and associated to a set of nodes $\left\{x_{j}\right\}_{j \in \mathcal{J}}$. Figure 1 presents an example. It shows a spatial domain where finite element nodes are considered only along the Dirichlet boundary. Those are the active nodes, $\left\{x_{j}\right\}_{j \in \mathcal{J}}$, for the functional interpolation. Other non-active nodes are considered to define the support of the finite element shape functions (thus only associated to the geometrical interpolation). 


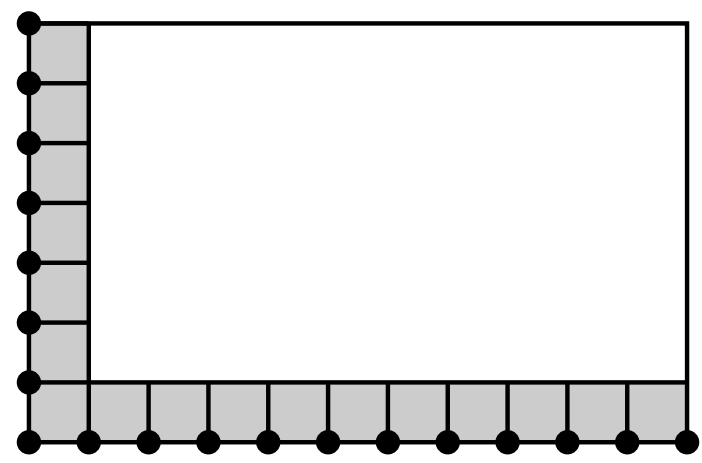

Fig. 1. Finite element active nodes $(\bullet)$ and support of the incomplete base of finite element shape functions (in gray).

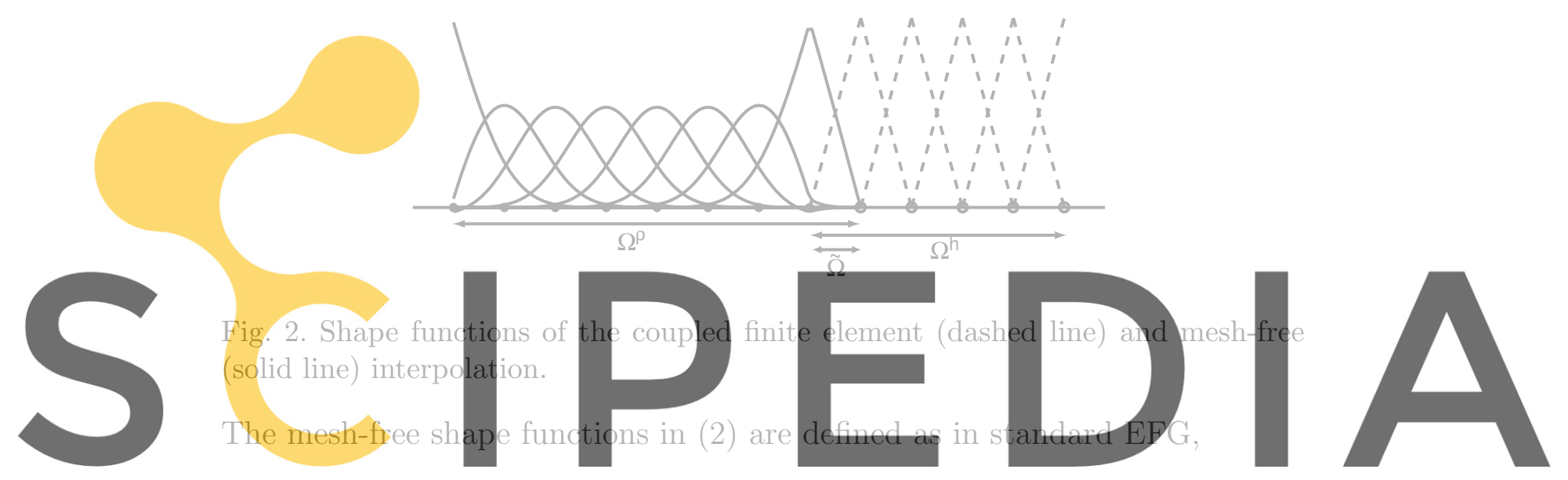

Register for free at https//www.scipedia.com $\widetilde{A}^{p}(x)$ downlpad the version without the watermark

but the unknown vector $\widetilde{\alpha}$ is determined imposing the reproducibility condition associated to the combined approximation (2), that is

$$
\mathbf{P}(x)=\pi^{h} \mathbf{P}(x)+\sum_{i \in \mathcal{I}} \widetilde{N}_{i}^{\rho}(x) \mathbf{P}\left(x_{i}\right)
$$

Substitution of (3) in (4) leads to a small system of equations for $\widetilde{\boldsymbol{\alpha}} \in \mathbb{R}^{l+1}$, see [12] for details,

$$
\mathbf{M}(x) \widetilde{\boldsymbol{\alpha}}(x)=\mathbf{P}(x)-\pi^{h} \mathbf{P}(x)
$$

The only difference with standard EFG is the modification of the r.h.s. of the previous system, in order to take into account the contribution of the finite element base in the approximation. In Figure 2 this is clearly shown, the meshfree shape functions adapt their shape to recover the linear interpolation.

Remark 2 In the region where only particles are present $\pi^{h} \mathbf{P}(x)=0$. Thus, in that region $\widetilde{\boldsymbol{\alpha}}=\boldsymbol{\alpha}$ and the mesh-free shape functions $\widetilde{N}_{i}^{\rho}$ defined by (3) coincide with the standard EFG shape functions $N_{i}^{\rho}$ defined by (1). 
The domain $\Omega$ can be divided in three non disjoint regions: one where finite elements have an influence, $\Omega^{h}$, another where particles have an influence, $\Omega^{\rho}$, and finally, one transition region, $\widetilde{\Omega}=\Omega^{h} \cap \Omega^{\rho}$, where both finite elements and particles take care of the interpolation, see Figure 2. In the region where only finite elements are present, $\Omega^{h} \backslash \Omega^{\rho}$, a standard finite element approximation is used, in the region where only particles have an influence, $\Omega^{\rho} \backslash \Omega^{h}$, the standard EFG approximation is considered, but in the area where both interpolations have an influence, $\widetilde{\Omega}$, the coupled interpolation (2) is used. It is important to note that continuity of the interpolation is ensured under some conditions, even in multiple dimensions, by the following result, see also [21].

Proposition 1 The approximation $\widetilde{u}(x)$ is continuous in $\Omega$ provided that:

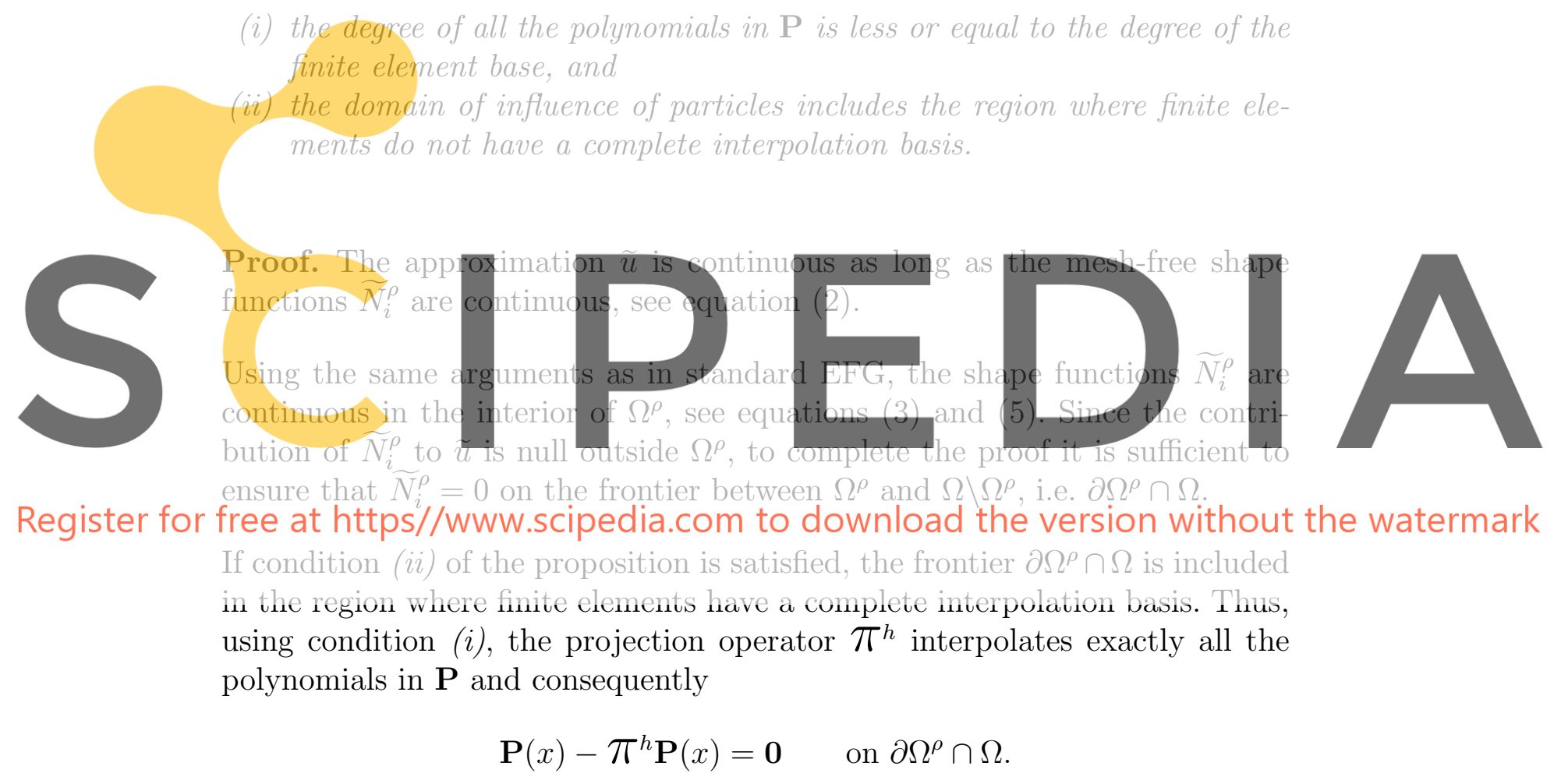

Recalling (5), the previous equation implies that $\widetilde{\boldsymbol{\alpha}}(x)=\mathbf{0}$, and consequently, $\widetilde{N}_{i}^{\rho}(x)=0$ on $\partial \Omega^{\rho} \cap \Omega$, as we wanted to prove.

In practice, the same order of consistency is imposed both for finite element and mesh-free interpolations (i.e. $m$ equal to the degree of the finite element base) and the domain of influence of particles coincides exactly with the region where finite elements do not have a complete interpolation basis.

In the regions where the finite element base is complete the contribution of the particles is zero. In particular, this means that $\widetilde{N}_{i}^{\rho}=0$ in the finite element edges (or faces in 3D) whose nodes are all in $\mathcal{J}$ (active nodes). This is an 

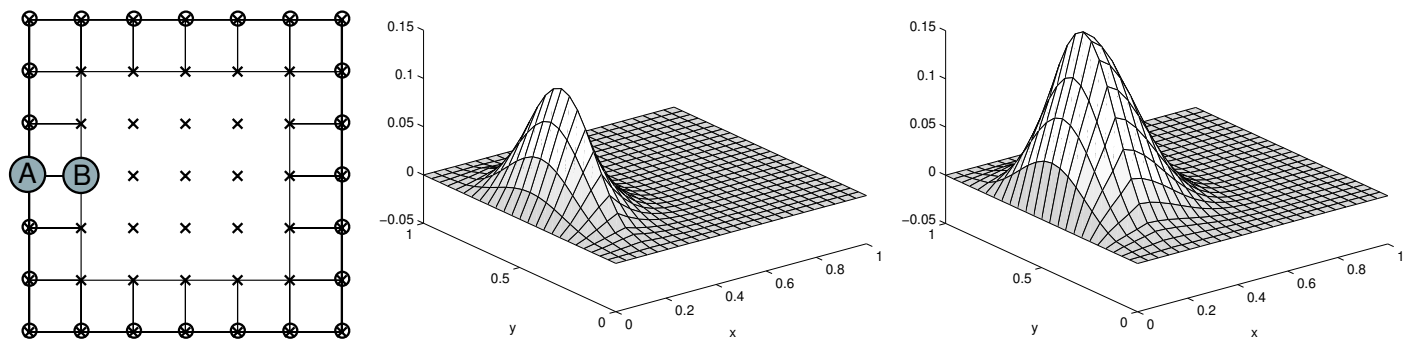

Fig. 3. Discretization with active finite element nodes at the boundary (o) and particles (x), and mesh-free shape function associated to the particle located at the gray circle (A) and (B) respectively.
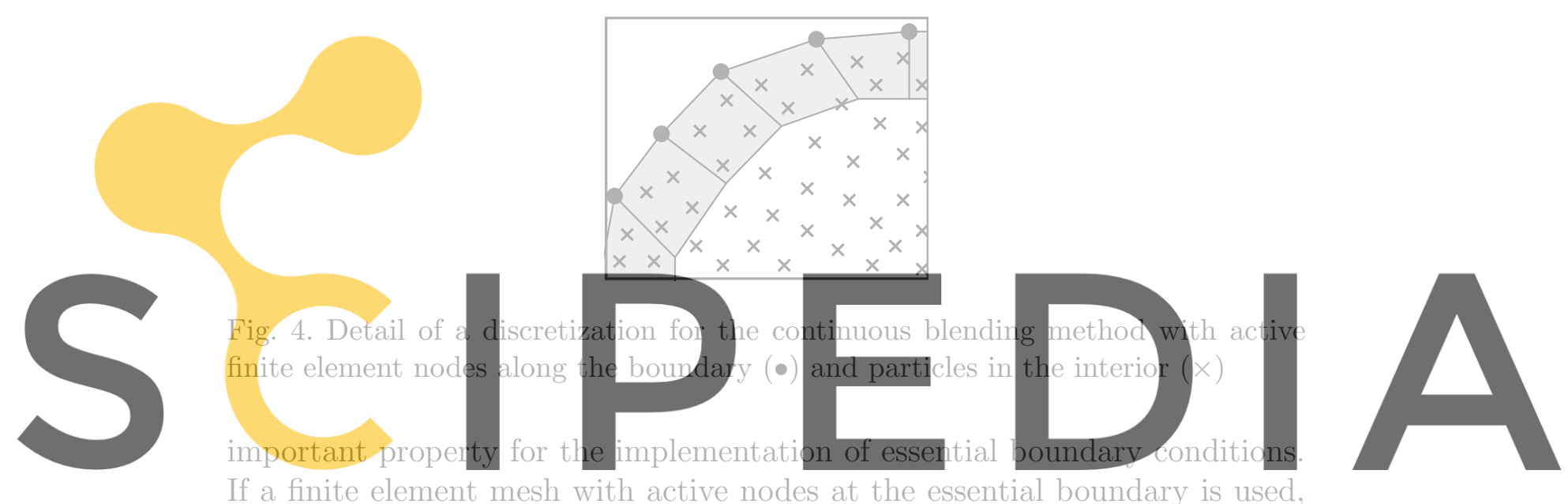

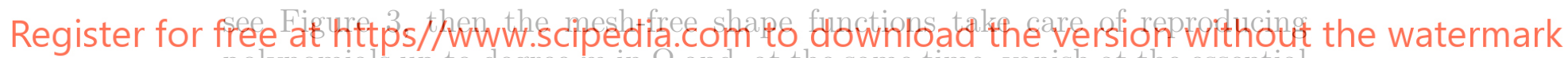
polynomials up to degree $m$ in $\Omega$ and, at the same time, vanish at the essential

boundary. Therefore, the prescribed values can be directly imposed as usual

in the framework of finite elements, just setting the value of the corresponding nodal coefficients. See Remark 3 in the next section for details.

Moreover, the continuous blending method allows the use of any distribution of particles. This is an important advantage with respect to the coupling of finite elements and EFG proposed in [11]. In this reference, the interior finite element nodes are replaced by particles and no more particles can be used in the region where finite elements have an influence. Figure 4 shows a possible discretization for the continuous blending method with active finite element nodes along the boundary. Note that, if desired, particles can be located also in the interior of the transition region (in gray). Thus, the distribution of particles can be as rich as needed near the boundary. This is of special importance in problems that require a rich interpolation near the boundary, such as mechanical problems with large deformations near the boundaries. For instance, in contact problems the interest of a finite element surface mesh is obvious and the advantage of enriching the area close to the boundary is crucial both for precision and large deformations. 


\section{Methods based on a modification of the weak form}

For the sake of clarity, the following model problem is considered

$$
\left\{\begin{aligned}
-\Delta u & =f & & \text { in } \Omega \\
u & =u_{d} & & \text { on } \Gamma_{d} \\
\nabla u \cdot \boldsymbol{n} & =g_{n} & & \text { on } \Gamma_{n}
\end{aligned}\right.
$$

where $\bar{\Gamma}_{d} \cup \bar{\Gamma}_{n}=\partial \Omega$ and $\boldsymbol{n}$ is the outward normal unit vector on $\partial \Omega$. The generalization of the following developments to other PDEs is straightforward.

The weak problem associated to (6) is "find $u \in \mathcal{H}^{1}(\Omega)$ such that $u=u_{d}$ on $\Gamma_{d}$ and

$$
\int_{\Omega} \nabla v \cdot \nabla u d \Omega-\int_{\Gamma_{d}} v \nabla u \cdot \boldsymbol{n} d \Gamma=\int_{\Omega} v f d \Omega+\int_{\Gamma_{n}} v g_{n} d \Gamma
$$

\section{for all $v \in \mathcal{H}^{1}(\Omega)$ ". In the framework of the finite element method, the inter-} polation of $u$ can easily be forced to verify the essential boundary condition,
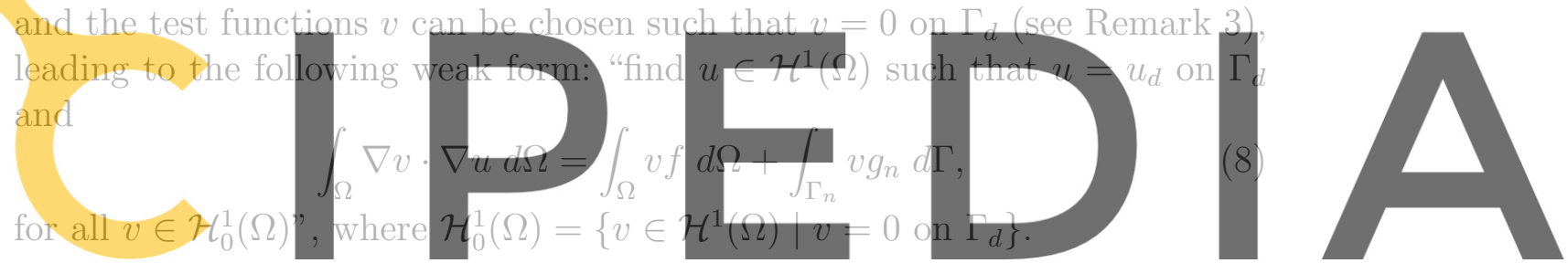

Remark 3 In the finite element method, or in the context of the continuous

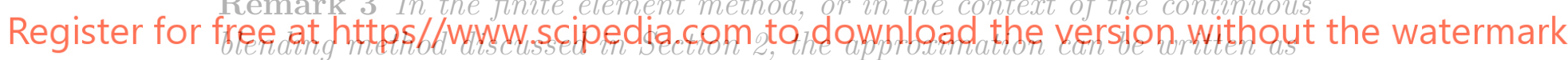

$$
u(x) \simeq \sum_{i \notin \mathcal{B}} u_{i} N_{i}(x)+\psi(x)
$$

where $\psi(x)=\sum_{j \in \mathcal{B}} u_{d}\left(x_{j}\right) N_{j}(x)$, and $\mathcal{B}$ is the set of indexes of all nodes on the essential boundary (nodes with prescribed value). In finite elements the shape functions are $N_{i}(x)=N_{i}^{h}(x)$; in the continuous blending method $N_{i}(x)=N_{i}^{h}(x)$ for $i$ corresponding to the finite element nodes (at least those in $\mathcal{B})$ and $N_{i}(x)=N_{i}^{\rho}(x)$ for $i$ corresponding to the particles. Thus, due to the Kronecker delta property along the boundary, $N_{i} \in \mathcal{H}_{0}^{1}(\Omega)$ for $i \notin \mathcal{B}$ and the approximation defined by (9) verifies $u=u_{d}$ at the nodes on the essential boundary. Therefore, approximation (9) and $v=N_{i}$, for $i \notin \mathcal{B}$, can be considered for the discretization of the weak form (8) leading to the following system of equations

$$
\mathbf{K u}=\mathbf{f},
$$

where

$$
\begin{aligned}
K_{i j} & =\int_{\Omega} \nabla N_{i} \cdot \nabla N_{j} d \Omega, \\
f_{i} & =\int_{\Omega} N_{i} f d \Omega+\int_{\Omega} N_{i} \psi d \Omega+\int_{\Gamma_{n}} N_{i} g_{n} d \Gamma,
\end{aligned}
$$


and $\mathbf{u}$ is the vector of coefficients $u_{i}$.

However, when standard mesh-free interpolation is used, the shape functions usually do not verify the Kronecker delta property. Therefore, imposing $u=u_{d}$ and $v=0$ on $\Gamma_{d}$ is not as straightforward as in finite elements or as in the continuous blending method, and the weak form defined by (8) cannot be used. This section presents three methods that overcome this problem: the Lagrange multiplier method, the penalty method and Nistche's method.

\subsection{Lagrange multiplier method}

The solution of problem (6) can also be obtained as the solution of a minimization problem with constraints: " $u$ minimizes the energy functional

$$
\Pi(v)=\frac{1}{2} \int_{\Omega} \nabla v \cdot \nabla v d \Omega-\int_{\Omega} v f d \Omega-\int_{\Gamma_{n}} v g_{n} d \Gamma
$$

and verifies the essential boundary conditions." That is,
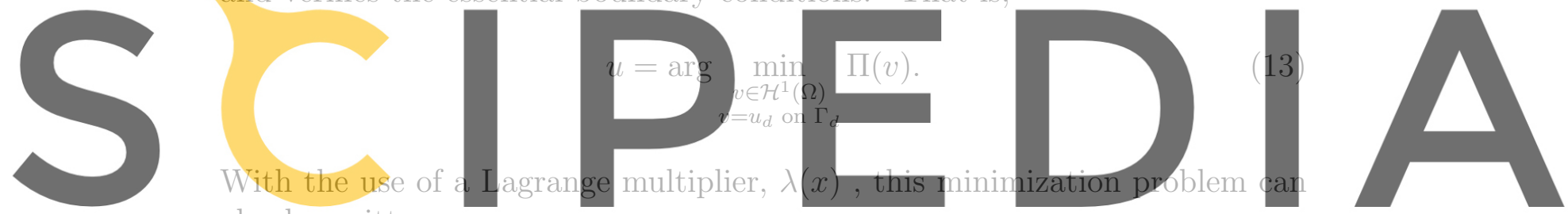

Register for free at https/ / www scipedia.com to fownlogad the versjion without the watermark

This min-max problem leads to the following weak form with Lagrange multiplier, "find $u \in \mathcal{H}^{1}(\Omega)$ and $\lambda \in \mathcal{H}^{-1 / 2}\left(\Gamma_{d}\right)$ such that

$$
\begin{array}{rlrl}
\int_{\Omega} \nabla v \cdot \nabla u d \Omega+\int_{\Gamma_{d}} v \lambda d \Gamma & =\int_{\Omega} v f d \Omega+\int_{\Gamma_{n}} v g_{n} d \Gamma, \forall v \in \mathcal{H}^{1}(\Omega) \\
\int_{\Gamma_{d}} \gamma\left(u-u_{d}\right) d \Gamma & =0, & \forall \gamma \in \mathcal{H}^{-\frac{1}{2}}\left(\Gamma_{d}\right) . "
\end{array}
$$

Remark 4 Equation (14b) imposes the essential boundary condition, $u=u_{d}$ on $\Gamma_{d}$, in weak form.

Remark 5 The physical interpretation of the Lagrange multiplier can be seen by simple comparison of equations (14a) and (7): the Lagrange multiplier corresponds to the flux (traction in a mechanical problem) along the essential boundary, $\lambda=-\nabla u \cdot \boldsymbol{n}$.

Considering now the approximation $u(x) \simeq \sum_{i} N_{i}(x) u_{i}$, with mesh-free shape functions $N_{i}$, and an interpolation for $\lambda$ with a set of boundary functions 


$$
\left\{N_{i}^{L}(x)\right\}_{i=1}^{\ell}
$$

$$
\lambda(x) \simeq \sum_{i=1}^{\ell} \lambda_{i} N_{i}^{L}(x) \quad \text { for } x \in \Gamma_{d}
$$

the discretization of (14) leads to the system of equations

$$
\left(\begin{array}{cc}
\mathbf{K} & \mathbf{A}^{T} \\
\mathbf{A} & 0
\end{array}\right)\left(\begin{array}{l}
\mathbf{u} \\
\boldsymbol{\lambda}
\end{array}\right)=\left(\begin{array}{l}
\mathbf{f} \\
\mathbf{b}
\end{array}\right),
$$

where $\mathbf{K}$ and $\mathbf{f}$ are already defined in (11) (use $\psi=0$ ), $\boldsymbol{\lambda}$ is the vector of coefficients $\lambda_{i}$, and

$$
A_{i j}=\int_{\Gamma_{d}} N_{i}^{L} N_{j} d \Gamma, \quad b_{i}=\int_{\Gamma_{d}} N_{i}^{L} u_{d} d \Gamma .
$$

There are several possibilities for the choice of the interpolation space for the Lagrange multiplier $\lambda$. Some of them are (1) a finite element interpolation on the essential boundary, (2) a mesh-free interpolation on the essential boundary or (3) the same shape functions used in the interpolation of $u$ restricted along $\Gamma_{d}$, i.e. $N_{i}^{L}=N_{i}$ for $i$ such that $\left.N_{i}\right|_{\Gamma_{d}} \neq 0$. However, the most popular choice is

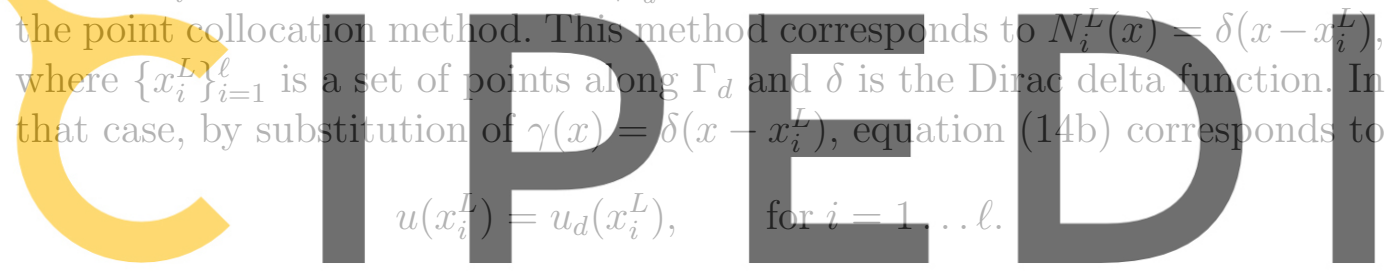

That is, $A_{i j}=N_{j}\left(x_{i}^{L}\right), b_{i}=u_{d}\left(x_{i}^{L}\right)$, and each equation of $\mathbf{A u}=\mathbf{b}$ in (16)

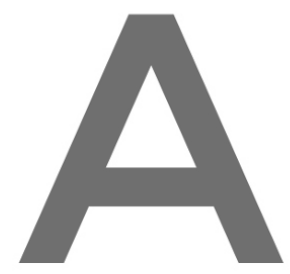

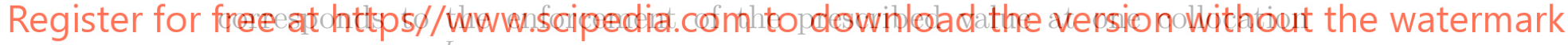
point, namely $x_{i}^{L}$.

The system of equations (16) can also be derived from the minimization in $\mathbb{R}^{\text {ndof }}$ of the discrete version of the energy functional (12),

$$
\widetilde{\Pi}(\mathbf{v})=\frac{1}{2} \mathbf{v}^{T} \mathbf{K} \mathbf{v}-\mathbf{f}^{T} \mathbf{v},
$$

subject to the constraints corresponding to the essential boundary conditions, $\mathbf{A u}=\mathbf{b}$. That is, by introduction of a vector multiplier $\boldsymbol{\gamma}$, the solution of (16) corresponds to

$$
(\mathbf{u}, \boldsymbol{\lambda})=\arg \min _{\mathbf{v} \in \mathbb{R}^{\text {nof }}} \max _{\in \mathbb{R}^{\ell}} \widetilde{\Pi}(\mathbf{v})+\boldsymbol{\gamma}^{T}(\mathbf{A v}-\mathbf{b}) .
$$

Therefore, the Lagrange multiplier method is, in principle, general and easily applicable to all kind of problems. In fact, there is no need to know the weak form with Lagrange multiplier, it is sufficient to define the discrete energy functional (17), i.e. compute $\mathbf{K}$ and $\mathbf{f}$, and the restrictions due to the boundary conditions, $\mathbf{A} \mathbf{u}=\mathbf{b}$, in order to determine the system of equations (16). However, the main disadvantages of the Lagrange multiplier method are: 
(1) The dimension of the resulting system of equations is increased.

(2) Even for $\mathbf{K}$ symmetric and semi-positive definite, the global matrix in (16) is symmetric but it is no longer positive definite. Therefore, standard linear solvers for symmetric and positive definite matrices can not be used.

(3) More crucial is the fact that the system (16) and the weak problem (14) induce a saddle point problem which precludes an arbitrary choice of the interpolation space for $u$ and $\lambda$. The discretization of the multiplier $\lambda$ must be accurate enough in order to obtain an acceptable solution, but the resulting system of equations turns out to be singular if the number of Lagrange multipliers $\lambda_{i}$ is too large. In fact, the interpolation spaces for the Lagrange multiplier $\lambda$ and for the principal unknown $u$ must verify an inf-sup condition, Babuska-Brezzi stability condition, in order to ensure the convergence of the approximation, see $[13,14]$ for details.

The first two disadvantages can be neglected in front of the versatility and straightforward implementation of the method. However, while in the finite element method it is trivial to choose the interpolation for the Lagrange multiplier in order to verify the Babuska-Brezzi stability condition and to impose accurate essential boundary conditions, this choice is not trivial for mesh-free methods. As it is shown in Section 4.2, in mesh-free methods the choice of an appropiate interpolation for the Lagrange multiplier can be a serious problem in particular situations.

\subsection{Penalty method}

The minimization problem with constraints defined by (13) can also be solved with the use of a penalty parameter. That is,

$$
u=\arg \min _{v \in \mathcal{H}^{1}(\Omega)} \Pi(v)+\frac{1}{2} \beta \int_{\Gamma_{d}}\left(v-u_{d}\right)^{2} d \Gamma .
$$

The penalty parameter $\beta$ is a positive scalar constant that must be large enough in order to impose the essential boundary condition with the desired accuracy. The minimization problem (18) leads to the following weak form: "find $u \in \mathcal{H}^{1}(\Omega)$ such that

$$
\int_{\Omega} \nabla v \cdot \nabla u d \Omega+\beta \int_{\Gamma_{d}} v u d \Gamma=\int_{\Omega} v f d \Omega+\int_{\Gamma_{n}} v g_{n} d \Gamma+\beta \int_{\Gamma_{d}} v u_{d} d \Gamma
$$

for all $v \in \mathcal{H}^{1}(\Omega)$ ". The discretization of this weak form leads to the system of equations

$$
\left(\mathbf{K}+\beta \mathbf{M}^{p}\right) \mathbf{u}=\mathbf{f}+\beta \mathbf{f}^{p},
$$

where $\mathbf{K}$ and $\mathbf{f}$ are defined in (11) (use $\psi=0$ ) and

$$
M_{i j}^{p}=\int_{\Gamma_{d}} N_{i} N_{j} d \Gamma, \quad f_{i}^{p}=\int_{\Gamma_{d}} N_{i} u_{d} d \Gamma .
$$


The penalty method can also be obtained from the minimization of the discrete version of the energy functional (17) in $\mathbb{R}^{\text {ndof }}$, subjected to the constraints corresponding to the essential boundary condition, $\mathbf{A u}=\mathbf{b}$. The discrete minimization problem is

$$
\mathbf{u}=\arg \min _{\mathbf{v} \in \mathbb{R}^{\text {ndof }}} \widetilde{\Pi}(\mathbf{v})+\frac{1}{2} \beta\|\mathbf{A v}-\mathbf{b}\|^{2},
$$

with the vector norm $\|\mathbf{x}\|^{2}=\mathbf{x}^{T} \mathbf{x}$. The solution of this minimization problem can be obtained as the solution of the linear system of equations

$$
\left(\mathbf{K}+\beta \mathbf{A}^{T} \mathbf{A}\right) \mathbf{u}=\mathbf{f}+\beta \mathbf{A}^{T} \mathbf{b} .
$$

Remark 6 If $\mathbf{A u}=\mathbf{b}$ is the set of constraints associated to the imposition of the essential boundary condition at $\ell$ points, $\left\{x_{k}^{p}\right\}_{k=1}^{\ell}$, then $A_{i j}=N_{j}\left(x_{i}^{p}\right)$ and $b_{i}=u_{d}\left(x_{i}^{p}\right)$. In that case, the coefficients of matrix $\mathbf{A}^{T} \mathbf{A}$ and vector $\mathbf{A}^{T} \mathbf{b}$ are

$$
\left[\mathbf{A}^{T} \mathbf{A}\right]_{i j}=\sum_{k=1}^{\ell} N_{i}\left(x_{k}^{p}\right) N_{j}\left(x_{k}^{p}\right), \quad\left[\mathbf{A}^{T} \mathbf{b}\right]_{i}=\sum_{k=1}^{\ell} N_{i}\left(x_{k}^{p}\right) u_{d}\left(x_{k}^{p}\right) .
$$

Therefore, matrix $\mathbf{A}^{T} \mathbf{A}$ and vector $\mathbf{A}^{T} \mathbf{b}$ in (21) can be interpreted as the approximation of matrix $\mathbf{M}^{p}$ and vector $\mathbf{f}^{p}$ in (20) using a numerical quadrature with integration points at $x_{k}^{p}$ and weights equal to one.

As previously observed with the Lagrange multiplier method, the penalty method is easily applicable to all kind of problems. The penalty method presents two clear advantages: (i) the dimension of the system is not increased and (ii) the matrix in the resulting system, see equation (20) or (21), is symmetric and positive definite, provided that $\mathbf{K}$ is symmetric and $\beta$ is large enough.

However, the penalty method has also two important drawbacks: the Dirichlet boundary condition is weakly imposed (the parameter $\beta$ controls how well the essential boundary condition is ensured) and the matrix in (20) is usually ill conditioned (the condition number increases with $\beta$ )

A general theorem on the convergence of the penalty method and the choice of parameter $\beta$ can be found in $[9,24]$. For an interpolation with consistency of order $p$ and discretization measure $h$ (i.e. the characteristic element size in finite elements or the characteristic distance between particles in a mesh-free method) the best error estimate obtained in [24] gives a rate of convergence of order $h^{\frac{2 p+1}{3}}$ in the energy norm, provided that the penalty $\beta$ is taken to be of order $h^{-\frac{2 p+1}{3}}$ [25]. In the linear case, it corresponds to the optimal rate of convergence in energy norm. For order $p \geq 2$, the lack of optimality in the rate of convergence is a direct consequence of the lack of consistency of the weak formulation, see [25] and Remark 7. The choice of the penalty $\beta$ to maintain 
the optimal rate of convergence in $\mathcal{L}^{2}$ norm and the ill-conditioning of the system are commented for a particular problem in Section 4.1.

\subsection{Nistche's method}

Nitsche's weak form for problem (6) is

$$
\begin{gathered}
\int_{\Omega} \nabla v \cdot \nabla u d \Omega-\int_{\Gamma_{d}} v \nabla u \cdot \boldsymbol{n} d \Gamma-\int_{\Gamma_{d}} u \nabla v \cdot \boldsymbol{n} d \Gamma+\beta \int_{\Gamma_{d}} v u d \Gamma \\
=\int_{\Omega} v f d \Omega+\int_{\Gamma_{n}} v g_{n} d \Gamma-\int_{\Gamma_{d}} u_{d} \nabla v \cdot \boldsymbol{n} d \Gamma+\beta \int_{\Gamma_{d}} v u_{d} d \Gamma
\end{gathered}
$$

where $\beta$ is a positive constant scalar parameter $[25,26]$.

Comparing with the weak form defined by (7), the new terms in the 1.h.s. of (22) are $\int_{\Gamma_{d}} u \nabla v \cdot \boldsymbol{n} d \Gamma$, which recovers the symmetry of the bilinear form, and $\beta \int_{\Gamma_{d}} v u d \Gamma$, that ensures the coercivity of the bilinear form (i.e. the matrix corresponding to its discretization is positive definite) provided that $\beta$ is large enough. The new terms in the r.h.s. are added to ensure consistency of the weak form.

The discretization of the Nitsche's weak form leads to a system of equations with the same size as $\mathbf{K}$ and whose matrix is symmetric and positive definite, provided that $\mathbf{K}$ is symmetric and $\beta$ is large enough. Although, as in the penalty method, the condition number of this matrix increases with parameter $\beta$, in practice not very large values are needed in order to ensure convergence and a proper implementation of the boundary condition (see examples in Section 4). The matrix condition number is not a real problem for this method.

Remark 7 Nitsche's method can be interpreted as a consistent improvement of the penalty method. The penalty weak form (19) is not consistent, in the sense that the solution of (6) does not verify the penalty weak form for trial test functions that do not vanish at $\Gamma_{d}$, see [25]. Nitsche's weak form keeps the term $\int_{\Gamma_{d}} v \nabla u \cdot \boldsymbol{n} d \Gamma$ from the consistent weak form (7), and includes new terms maintaining the consistency.

The only problem of Nitsche's method is the deduction of the weak form. The generalization of the implementation for other problems is not as straightforward as for the method of Lagrange multipliers or for the penalty method. The weak form and the choice of parameter $\beta$ depends not only on the partial differential equation, but also on the essential boundary condition to be prescribed. Nitsche's method applied to other problems can be found in [26], in [27] for the Navier-Stokes problem, in [28] for the Stokes problem, or in [29] 
for elasticity problems.

Regarding the choice of the parameter, Nitsche proved that if $\beta$ is taken as $\beta=\alpha / h$, where $\alpha$ is a large enough constant and $h$ denotes the discretization characteristic measure, then the discrete solution converges to the exact solution with optimal order in $\mathcal{H}^{1}$ and $\mathcal{L}^{2}$ norms. Moreover, for model problem (6) with Dirichlet boundary conditions, $\Gamma_{d}=\partial \Omega$, a value for constant $\alpha$ can be determined taking into account that convergence is ensured if $\beta>2 C^{2}$, where $C$ is a positive constant such that $\|\nabla v \cdot \boldsymbol{n}\|_{\mathcal{L}^{2}(\partial \Omega)} \leq C\|\nabla v\|_{\mathcal{L}^{2}(\Omega)}$ for all $v$ in the chosen interpolation space. This condition ensures the coercivity of the bilinear form in the interpolation space. In a recent paper [8], Griebel and coworkers propose the estimation of constant $C$ as the maximum eigenvalue of the generalized eigenvalue problem,

$$
\mathbf{A v}=\lambda \mathbf{B v}
$$

where

$$
A_{i j}=\int_{\partial \Omega}\left(\nabla N_{i} \cdot \boldsymbol{n}\right)\left(\nabla N_{j} \cdot \boldsymbol{n}\right) d \Gamma, \quad B_{i j}=\int_{\Omega} \nabla N_{i} \cdot \nabla N_{j} d \Omega
$$

\section{Numerical examples}

Two 2D numerical examples are used to compare the methods described in previous sections for the imposition of essential boundary conditions: a Laplace problem with known analytical solution, and a linear elasticity problem with a discontinuous boundary condition. The EFG method with bilinear consistency and $\rho \simeq 3.2 h$, where $h$ denotes the distance between particles, is used in all examples. However, similar results can be obtained with other mesh-free methods based on a Galerkin weak form.

\subsection{Laplace equation}

The 2D Laplace problem

$$
\left\{\begin{array}{l}
\Delta u=0 \quad(x, y) \in] 0,1[\times] 0,1[ \\
u(x, 0)=\sin (\pi x) \\
u(x, 1)=u(0, y)=u(1, y)=0
\end{array}\right.
$$

with known analytical solution [17],

$$
u(x, y)=[\cosh (\pi y)-\operatorname{coth}(\pi y) \sinh (\pi y)] \sin (\pi x),
$$



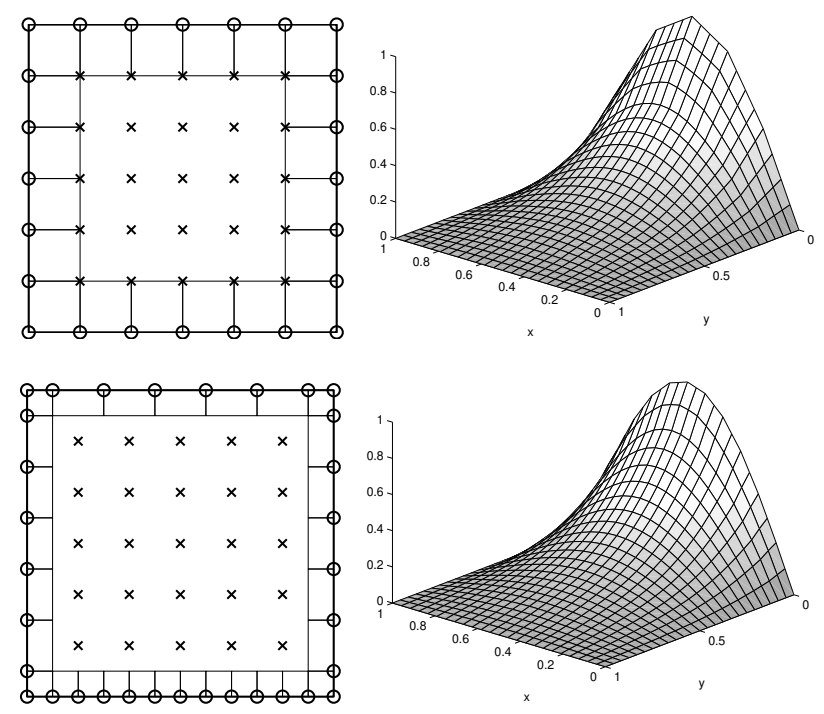

Fig. 5. Continuous blending of EFG with finite elements: particles are marked with $\times$ and nodes are marked with $\mathrm{o}$

is considered next.

Figure 5 shows the solution obtained for the continuous blending of EFG with bilinear finite elements. The discretization is also represented: circles indicate active nodes and crosses indicate particles. In every example the distance between particles is $h=1 / 6$, but a finer mesh is used for the representation of the solution. Two different finite element discretizations at the boundary are considered. In the coarse one shown in Figure 5 (top) the linear finite element interpolation at the boundary can be clearly observed. In the refined one in Figure 5 (bottom), the approximation of the boundary condition is improved using smaller finite elements along $\{y=0\} \cap \partial \Omega$. As noted in Remark 3 , the imposition of essential boundary conditions is trivial in the continuous blending method; moreover, it is also easy to control the error along this boundary.
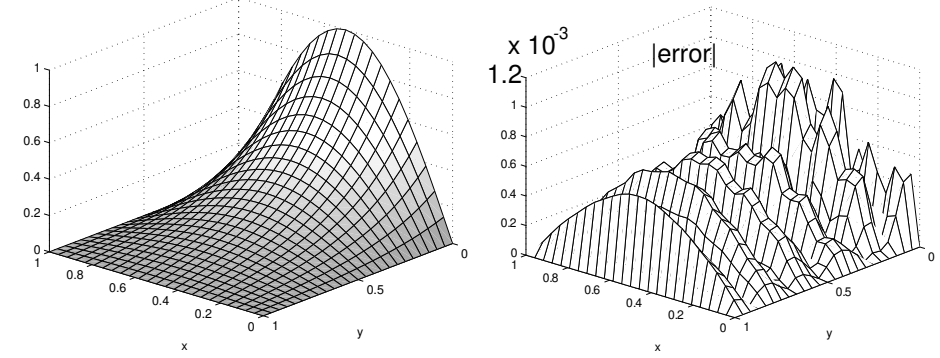

Fig. 6. Solution (left) and absolute value of the error (right) for the Lagrange multiplier method

Figures 6, 7 and 9 show the solution obtained with methods based on a modification of the weak form. In all cases a distribution of $7 \times 7$ particles is 
considered, i.e. the distance between particles is $h=1 / 6$.

Figure 6 shows the solution and the absolute value of the error for the EFG method with Lagrange multipliers. The essential boundary condition is imposed by collocation at the particles located on the boundary. Although the error is not zero along this boundary (it is zero only at the collocation points), an accurate solution is obtained in the whole domain.
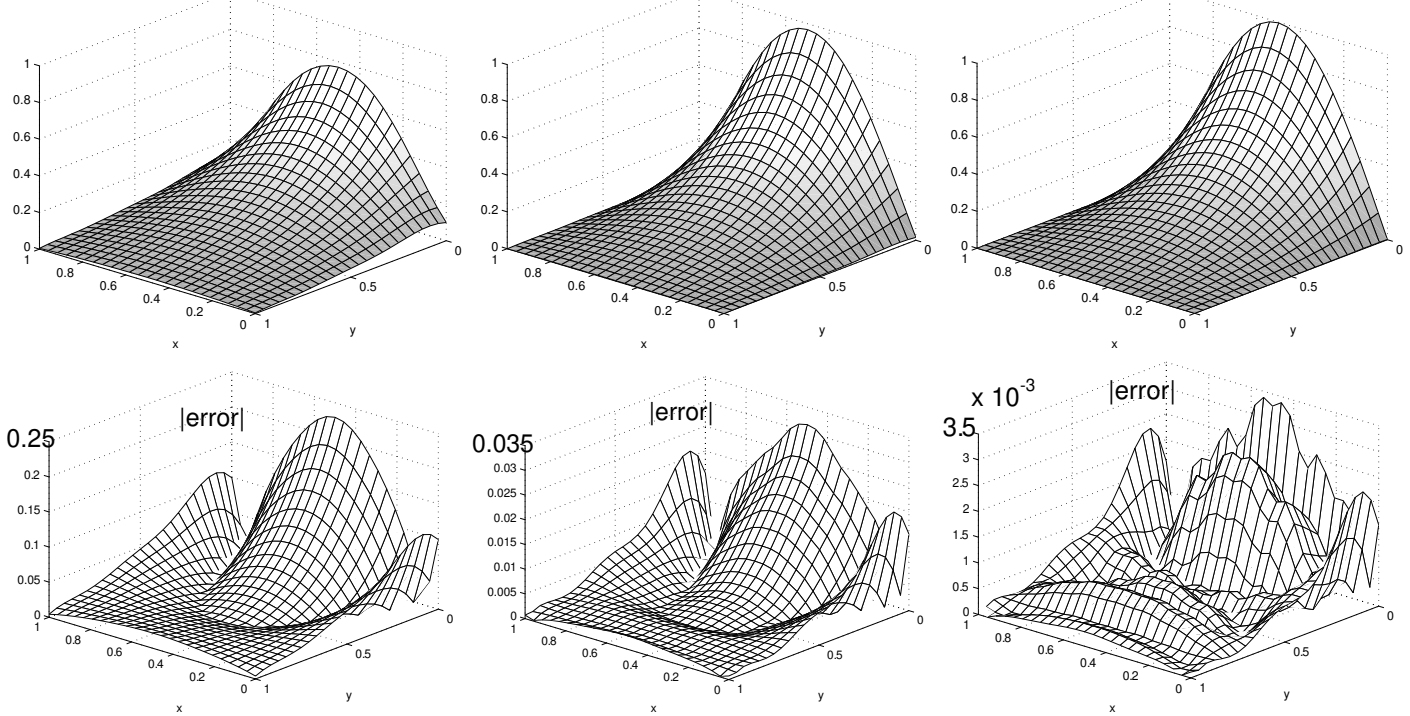

Fig. 7. Penalty method solution (top) and error (bottom) for $\beta=10$ (left), $\beta=100$ (center) and $\beta=10^{3}$ (right)
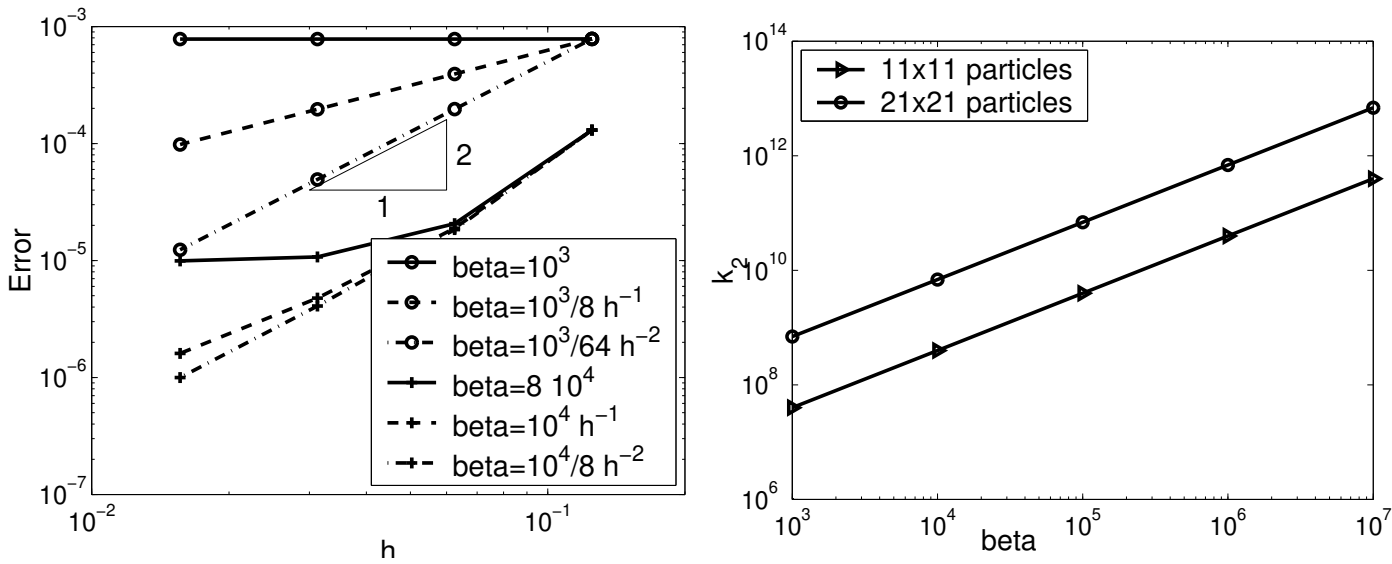

Fig. 8. Evolution of the $\mathcal{L}^{2}(\Omega)$ error norm for the penalty method and matrix condition number

The behavior of the penalty method is analyzed next. Figure 7 shows the solution for increasing values of the penalty parameter $\beta$. The penalty parameter must be large enough, $\beta \geq 10^{3}$, in order to impose the boundary condition in an accurate manner. Figure 8 shows convergence curves for different choices of the penalty parameter. The penalty method converges with a rate close to 2 in the $\mathcal{L}^{2}$ norm if the penalty parameter $\beta$ is proportional to $h^{-2}$. If the penalty 
parameter is constant, or proportional to $h^{-1}$, the boundary error dominates and the optimal convergence rate is lost as $h$ goes to zero.

Figure 8 also shows the matrix condition number for increasing values of the penalty parameter, for a distribution of $11 \times 11$ and $21 \times 21$ particles. The condition number grows linearly with the penalty parameter. Note that, for instance, for a discretization with $21 \times 21$ a reasonable value for the penalty parameter is $\beta=10^{6}$ which corresponds to a condition number near $10^{12}$. Obviously, the situation gets worse for denser discretizations, which need larger penalty parameters. The ill-conditioning of the matrix reduces the applicability of the penalty method.
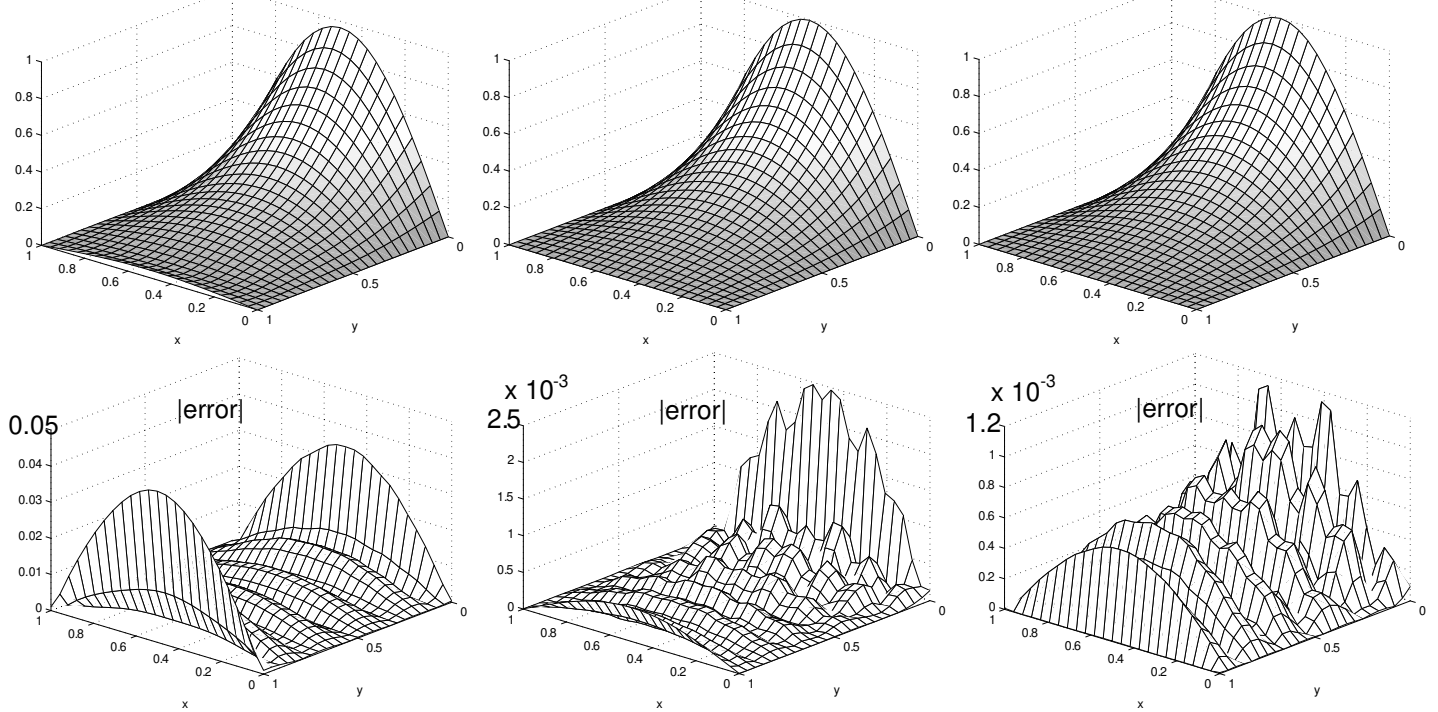

Fig. 9. Nitsche's method solution (top) and error (bottom) for $\beta=20$ (left), $\beta=55$ (eigenvalue estimate) and $\beta=10^{4}$ (right)

Figure 9 shows the approximation for Nitsche's method with different values of $\beta$. The value $\beta=55$ has been calculated as the maximum eigenvalue of (23). Smaller values, such as $\beta=20$, can lead to unacceptable solutions. However, the boundary condition is also properly imposed for $\beta>55$.

With a $7 \times 7$ distribution of particles, all the methods based on a modification of the weak form lead to solutions with similar accuracy. Regarding convergence, Figure 10 shows a comparison of the convergence for all methods. The first discretization pattern in Figure 5 is used for the continuous blending method. The penalty method uses the best penalty parameter observed in Figure 8, i.e. $\beta=\frac{10^{4}}{8} h^{-2}$. Finally, Nitsche's method is shown for the parameter proposed by the eigenvalue problem (23) (proportional to $h^{-1}$ ). As expected, the convergence rate is near 2 for all methods and all methods based on a modification of the weak form provide similar accuracy. The results for the continuous blending method are less accurate because of the interpolation of the boundary condition with linear finite elements. In fact the error is 

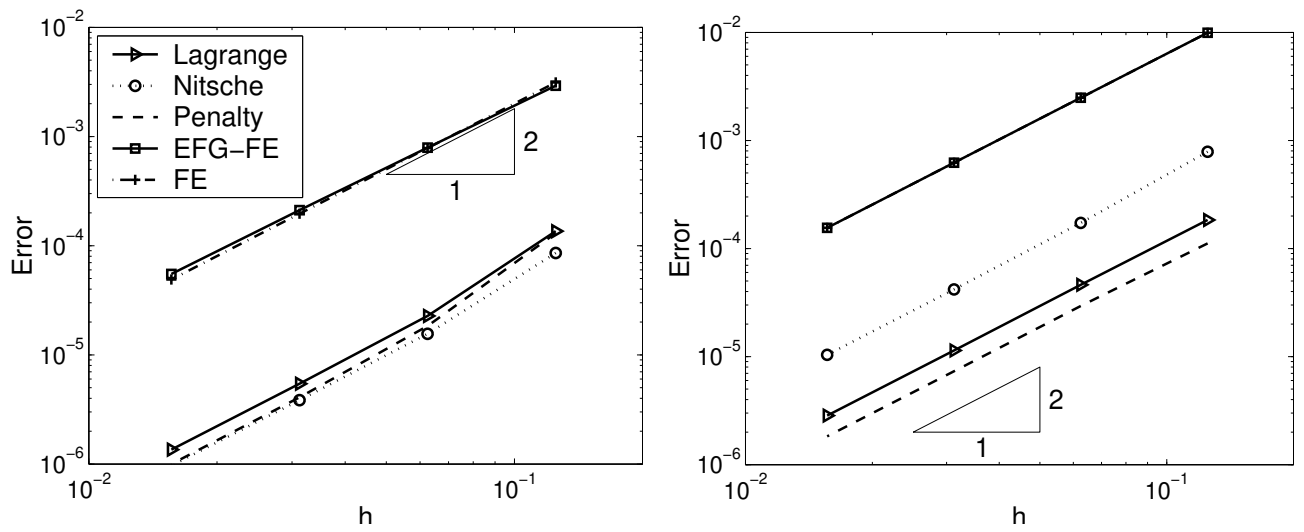

Fig. 10. Comparison of the $\mathcal{L}^{2}(\Omega)$ (left) and $\mathcal{L}^{2}(\partial \Omega)$ (right) error norms.

similar to the error with bilinear finite elements in the whole domain. That is, the error of the continuous blending method is dominated by the finite element error. Better results could be obtained with a discretization similar to the second discretization shown in Figure 5.

In conclusion, on one hand, the major advantage of coupling a mesh-free approximation with finite elements is the direct enforcement of the prescribed values. The accuracy of the approximation depends on both, the distribution of particles and the finite element discretization near the boundary. On the other hand, methods based on a modification of the weak form allow the use of the original mesh-free shape functions. The applicability of the penalty method is reduced due to the possible ill-conditioning problems, specially when refined discretizations are needed. The Lagrange multiplier method and the penalty method present similar properties. The advantage of Nitsche's method is that it requires only the choice of a scalar parameter, in front of the choice of the interpolation space for the Lagrange multiplier. For instance, the choice of the position of the collocation points in the Lagrange multiplier method can be a difficult task for irregular distributions of particles. However, it is fair to recall that the Lagrange multiplier method is easily applicable for the implementation of all sort of linear boundary constraints in a large variety of problems.

\subsection{Elasticity problem}

The resolution of the 2D linear elasticity problem represented in Figure 11 is considered in this section. Figure 11 also shows the solution obtained with a regular mesh of $30 \times 30$ biquadratic finite elements. The resolution with the EFG method is considered next in order to analyze the behavior of the continuous blending of EFG with finite elements, the Lagrange multiplier method and Nitsche's method. In all figures the distance between particles is $h=1 / 6$ 


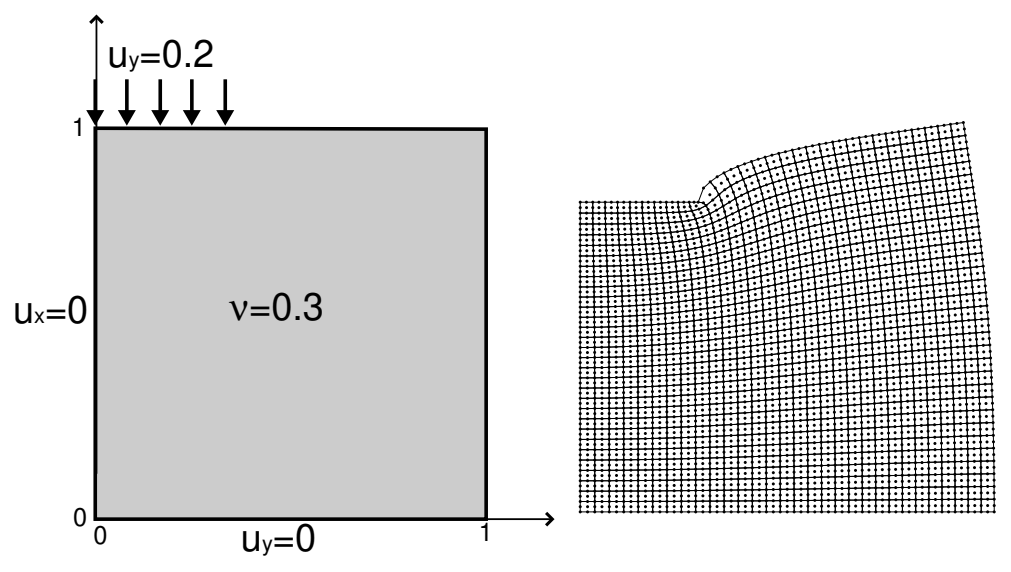

Fig. 11. Problem statement and solution with $30 \times 30$ biquadratic finite elements $(61 \times 61=3721$ nodes $)$
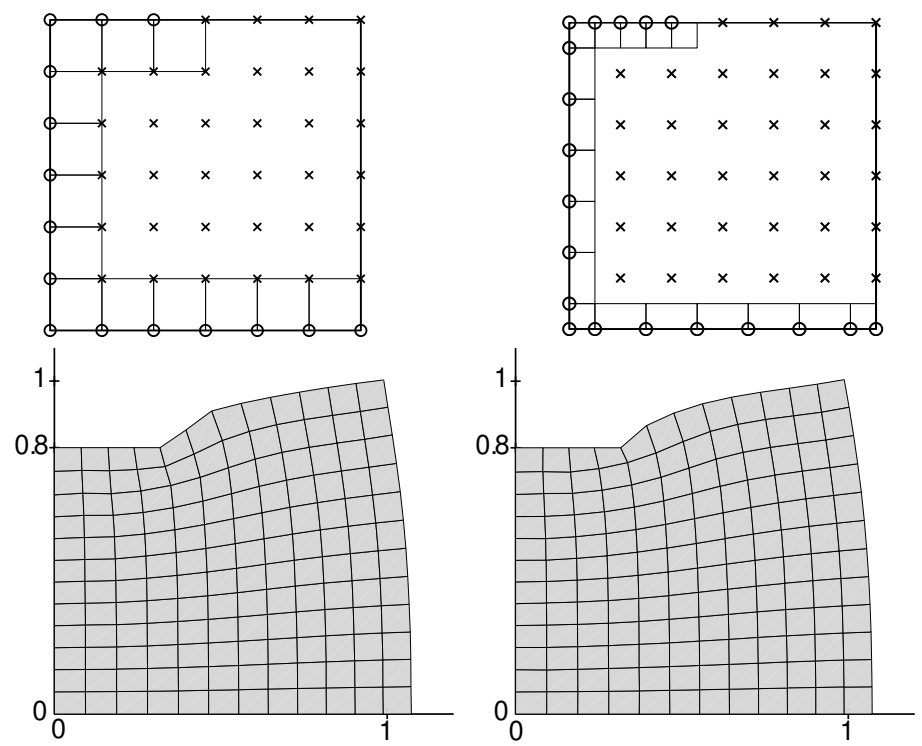

Fig. 12. Continuous blending with finite elements for two different distributions of finite elements near the essential boundary and the same distribution of particles, $h=1 / 6$

and a finer mesh is used for the representation of the solution.

Figure 12 shows the solution obtained coupling the EFG interpolation with linear finite elements. As observed in Remark 3, the prescribed displacements are directly imposed, i.e. the value of the corresponding nodal coefficient is set to the prescribed value. Two different finite element discretizations are considered. In both cases, the linear finite element approximation at the boundary, allows the exact enforcement of the prescribed displacement. Note that if the prescribed displacement is piecewise linear or piecewise constant, as it is in this example, then it is imposed exactly when a bilinear finite element approximation is used. The second discretization reduces the region of influence of the finite element shape functions. Therefore, the standard EFG approxima- 

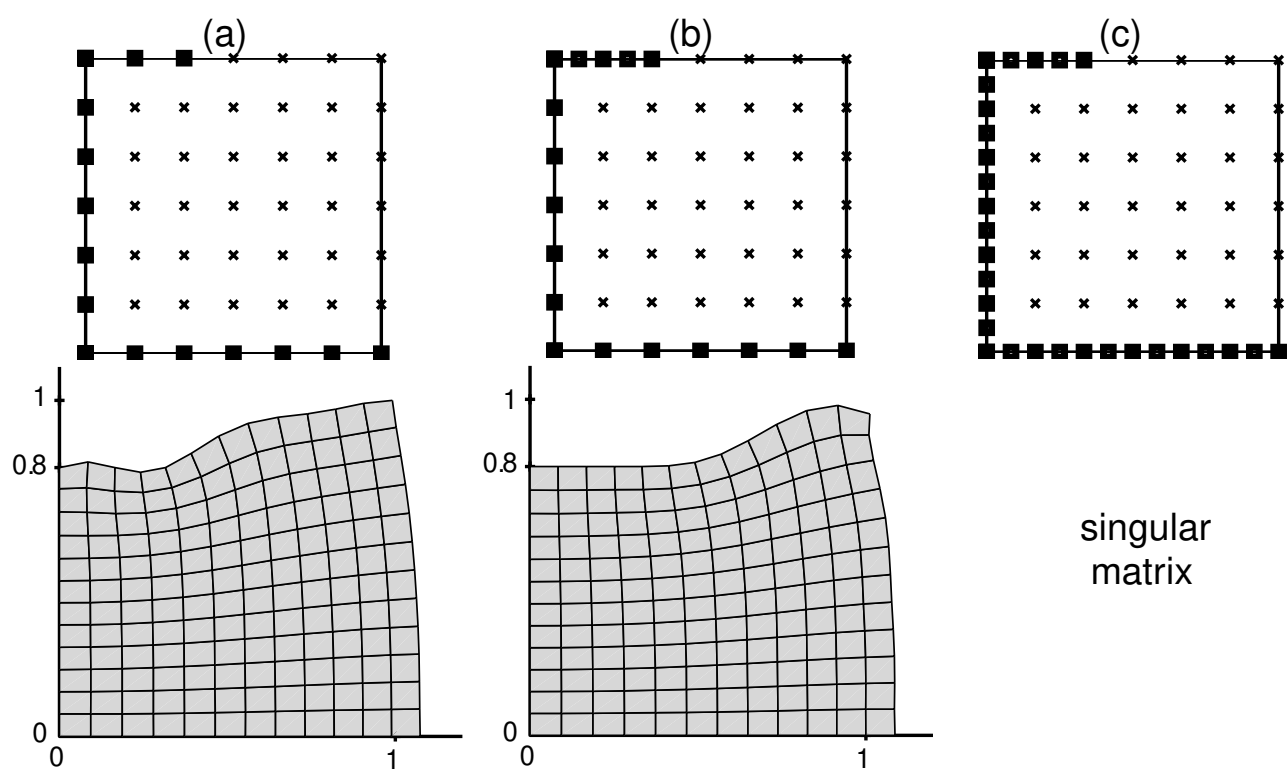

Fig. 13. Solution with Lagrange multipliers for three possible distributions of collocation points (black squares) and $7 \times 7$ particles

tion, usually with more precision and smoothness than finite elements, is used in a larger region.

Figures 13, 14 and 15 show the results obtained with methods that modify the weak form. Figures 13 and 14 show the solution obtained for the Lagrange multiplier method with different choices of the interpolation of the Lagrange multiplier. In Figure 13 the prescribed displacement is imposed at some collocation points, $x_{i}^{L}$, at the essential boundary (marked with black squares). Three possible distributions for the collocation points are considered. In the first one the collocation points correspond to the particles located at the essential boundary. The prescribed displacement is exactly imposed at the collocation points, but not along the rest of the essential boundary. Note that the displacement field is not accurate because of the smoothness of the mesh-free interpolation. But if the number of collocation points is too large the inf-sup condition is no longer verified and a singular matrix is obtained. This is the case of discretization (c) which corresponds to double the density of collocation points along the essential boundary. In this example, the choice of a proper interpolation for the Lagrange multiplier is not trivial. Option (b) represents a distribution of collocation points that imposes the prescribed displacements in a correct manner and, at the same time, leads to a regular matrix. Similar results are obtained if the Lagrange multiplier is interpolated with boundary linear finite elements, see Figure 14.

Therefore, although imposing boundary constraints is straightforward with the Lagrange multiplier method, the applicability of this method in particular cases can be clearly reduced due to the difficulty in the selection of a proper in- 

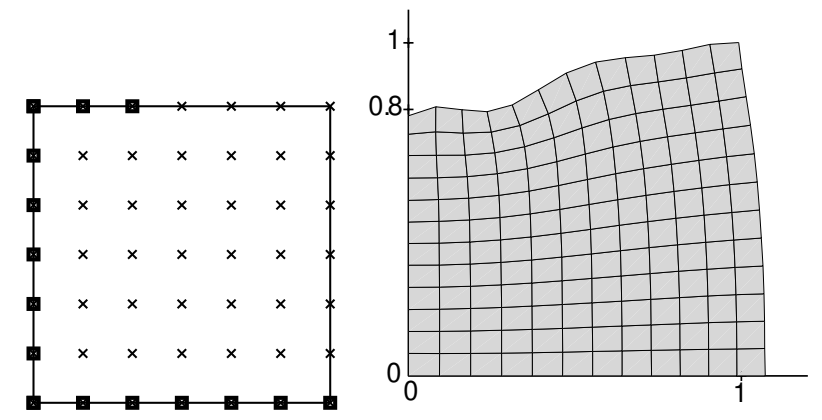

Fig. 14. Solution using boundary linear finite elements for the interpolation of the Lagrange multiplier
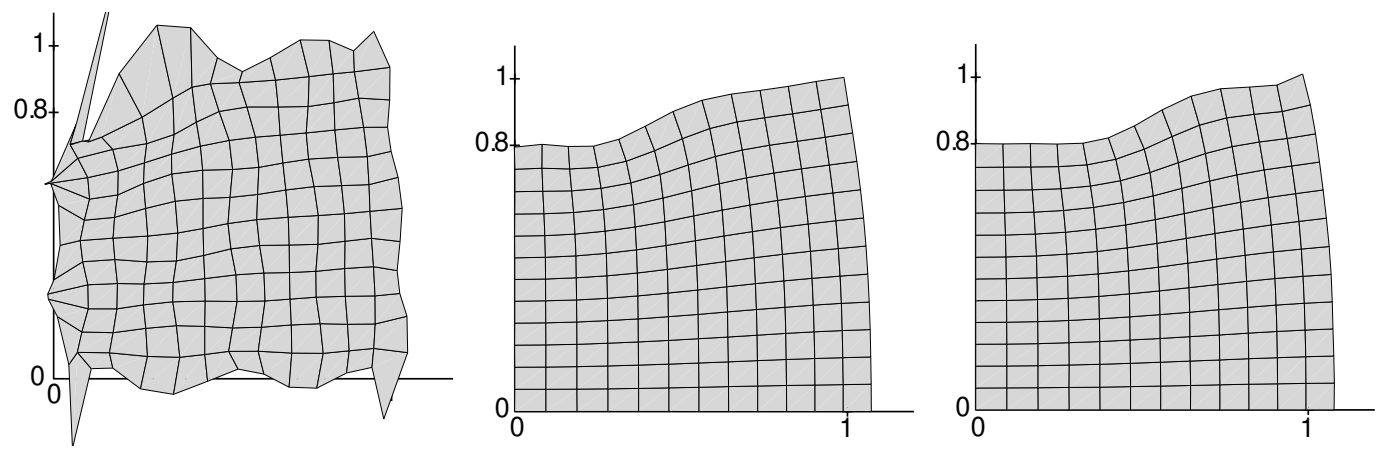

Fig. 15. Nitsche's solution with a $7 \times 7$ distribution of particles for $\beta=10$ (left), $\beta=100$ (center) and $\beta=10^{4}$ (right)

terpolation space for the Lagrange multiplier. It is important to note that the choice of the interpolation space can be even more complicated if an irregular distribution of particles is used. In that situation, Nitsche's method represents an interesting alternative for the weak imposition of essential boundary conditions.

The problem described in Figure 11 can be formalized as

$$
\begin{cases}\nabla \cdot \boldsymbol{\sigma}(\boldsymbol{u})=\mathbf{0} & \text { in } \Omega \\ \boldsymbol{\sigma}(\boldsymbol{u}) \cdot \boldsymbol{n}=0 & \text { on } \Gamma_{n} \\ \boldsymbol{u} \cdot \boldsymbol{n}=g_{d},(\boldsymbol{\sigma}(\boldsymbol{u}) \cdot \boldsymbol{n}) \cdot \boldsymbol{\tau}=0 & \text { on } \Gamma_{d}\end{cases}
$$

where $\boldsymbol{u}$ is the displacement vector, $\boldsymbol{\sigma}(\boldsymbol{u})$ is the corresponding stress, $\partial \Omega=$ $\bar{\Gamma}_{d} \cup \bar{\Gamma}_{n}, \boldsymbol{n}$ is the unit outward normal vector on $\partial \Omega, \boldsymbol{\tau}$ is the unit tangent vector, $\boldsymbol{\tau} \cdot \boldsymbol{n}=0$, and $g_{d}$ is the prescribed displacement. Nitsche's weak form of this linear elasticity problem is

$$
\begin{gathered}
\int_{\Omega} \boldsymbol{\varepsilon}(\boldsymbol{v}): \boldsymbol{\sigma}(\boldsymbol{u}) d \Omega-\int_{\Gamma_{d}}(\boldsymbol{v} \cdot \boldsymbol{n})(\boldsymbol{n} \cdot \boldsymbol{\sigma}(\boldsymbol{u}) \cdot \boldsymbol{n}) d \Gamma-\int_{\Gamma_{d}}(\boldsymbol{u} \cdot \boldsymbol{n})(\boldsymbol{n} \cdot \boldsymbol{\sigma}(\boldsymbol{v}) \cdot \boldsymbol{n}) d \Gamma \\
\quad+\beta \int_{\Gamma_{d}}(\boldsymbol{v} \cdot \boldsymbol{n})(\boldsymbol{u} \cdot \boldsymbol{n}) d \Gamma=-\int_{\Gamma_{d}} g_{d}(\boldsymbol{n} \cdot \boldsymbol{\sigma}(\boldsymbol{v}) \cdot \boldsymbol{n}) d \Gamma+\beta \int_{\Gamma_{d}} g_{d}(\boldsymbol{v} \cdot \boldsymbol{n}) d \Gamma
\end{gathered}
$$

for all $\boldsymbol{v} \in\left[\mathcal{H}^{1}(\Omega)\right]^{\text {nsd }}$, where $\boldsymbol{\varepsilon}(\boldsymbol{v})$ is the strain tensor associated to the dis- 
placement $\boldsymbol{v}$, and $\beta$ is a large enough constant which ensures the coercivity of the bilinear form. Figure 15 shows the solution obtained with Nitsche's method for different values of $\beta$. As in the previous example, small values of $\beta$, for instance $\beta=10$, can lead to unacceptable solutions. However, moderate values such as $\beta=100$ provide good results. For increasing values, $\beta$ plays the role of a penalty parameter, giving more weight to the verification of the boundary condition and, therefore, affecting to the solution in the rest of the domain. The great advantage of Nitsche's method is that parametric tuning can be done with only one scalar parameter $\beta$, in front of the difficult choice of the interpolation space for the Lagrange multiplier.

\section{Concluding remarks}

With the continuous blending method, which couples mesh-free and finite element methods, prescribed values can be directly enforced. This implies the modification of the mesh-free code in order to include finite elements, but the modifications are only made at the interpolation level. The accuracy of the approximation depends on the distribution of particles and also on the finite element discretization near the boundary. It is an efficient, robust and general purpose technique for imposing essential boundary conditions in mesh-free methods.

Methods based on a modification of the weak form, such as the Lagrange multiplier method, the penalty method and Nitsche's method, allow the use of standard mesh-free shape functions. The Lagrange multiplier method is one of the most popular, because of its straightforward implementation and applicability to a large variety of problems. However, attention must be paid to the choice of the interpolation space for the Lagrange multiplier. The discretization of the Lagrange multiplier must be accurate enough in order to obtain an acceptable solution, but it can lead to singular matrices if the interpolation space does not verify the Babuska-Brezzi stability condition. A simple 2D linear elasticity problem shows the major difficulties in the practical choice of the interpolation of the multiplier in particular situations. The penalty method and Nitsche's method require only the choice of one scalar parameter. The applicability of the penalty method is reduced due to the ill-conditioning of the resulting matrix and the lack of consistency of the weak formulation. As an alternative, Nitsche's method introduces new terms in the weak form in order to maintain consistency and coercivity of the bilinear form. Moreover, moderate values of the scalar parameter $\beta$ provide good results, avoiding the ill-conditioning problem of the penalty method. Therefore, Nitsche's method represents an interesting alternative to the widely used Lagrange multiplier method, mainly in those problems where the selection of an appropiate interpolation for the multiplier turns out to be a serious problem. 


\section{References}

[1] T. Belytschko, Y. Krongauz, D. Organ, M. Fleming, P. Krysl, Meshless methods: an overview and recent developments, Comput. Methods Appl. Mech. Eng. 139 (1-4) (1996) 3-47.

[2] W. K. Liu, T. Belytschko, J. T. Oden, editors, Meshless methods, Comput. Methods Appl. Mech. Eng. 139 (1-4) (1996) 1-440.

[3] W. K. Liu, Y. Chen, S. Jun, J. S. Chen, T. Belytschko, C. Pan, R. A. Uras, C. T. Chang, Overview and applications of the reproducing kernel particle methods, Arch. Comput. Methods Engrg. 3 (1) (1996) 3-80.

[4] T. Belytschko, Y. Y. Lu, L. Gu, Element free galerkin methods, Int. J. Numer. Methods Eng. 37 (2) (1994) 229-256.

[5] W. K. Liu, S. Jun, Y. F. Zhang, Reproducing kernel particle methods, Int. J. Numer. Methods Fluids 20 (8-9) (1995) 1081-1106.

[6] J. Bonet, S. Kulasegaram, Correction and stabilization of smooth particle hydrodynamics methods with applications in metal forming simulations, Int. J. Numer. Methods Eng. 47 (6) (2000) 1189-1214.

[7] T. Zhu, S. N. Atluri, A modified collocation method and a penalty formulation for enforcing the essential boundary conditions in the element free Galerkin method, Comput. Mech. 21 (3) (1998) 211-222.

[8] M. Griebel, M. A. Schweitzer, A particle-partition of unity method. Part V: Boundary conditions, in: S. Hildebrandt, H. Karcher (Eds.), Geometric Analysis and Nonlinear Partial Differential Equations, Springer, Berlin, 2002, pp. 517540 .

[9] I. Babuska, U. Banerjee, J. E. Osborn, Meshless and generalized finite element methods: A survey of some major results, in: M. Griebel, M. A. Schweitzer (Eds.), Meshfree methods for partial differential equations, Vol. 26 of Lecture Notes in Computational Science and Engineering, Springer-Verlag, Berlin, 2002, pp. 1-20, papers from the International workshop, Universität Bonn, Germany, September 11-14, 2001.

[10] J. Gosz, W. K. Liu, Admissible approximations for essential boundary conditions in the reproducing kernel particle method, Comput. Mech. 19 (2) (1996) 120-135.

[11] T. Belytschko, D. Organ, Y. Krongauz, A coupled finite element-element-free Galerkin method, Comput. Mech. 17 (3) (1995) 186-195.

[12] A. Huerta, S. Fernández-Méndez, Enrichment and coupling of the finite element and meshless methods, Int. J. Numer. Methods Eng. 48 (11) (2000) 1615-1636.

[13] I. Babuska, The finite element method with lagrange multipliers, Numer. Math. 20 (1973) 179-192. 
[14] F. Brezzi, On the existence, uniqueness and approximation of saddle-point problems arising from Lagrangian multipliers, Rev. Française Automat. Informat. Recherche Opérationnelle Sér. Rouge 8 (R-2) (1974) 129-151.

[15] J. S. Chen, C. Pan, C. T. Wu, W. K. Liu, Reproducing kernel particle methods for large deformation analysis of non-linear, Comput. Methods Appl. Mech. Eng. 139 (1-4) (1996) 195-227.

[16] F. C. Günter, W. K. Liu, Implementation of boundary conditions for meshless methods, Comput. Methods Appl. Mech. Eng. 163 (1-4) (1998) 205-230.

[17] G. J. Wagner, W. K. Liu, Application of essential boundary conditions in meshfree methods: a corrected collocation method, Int. J. Numer. Methods Eng. 47 (8) (2000) 1367-1379.

[18] J.-S. Chen, W. Han, Y. You, X. Meng, A reproducing kernel method with nodal interpolation property, Int. J. Numer. Methods Eng. 56 (7) (2003) 935-960.

[19] A. Huerta, S. Fernández-Méndez, W. K. Liu, A comparison of two formulations to blend finite elements and mesh-free methods, Comput. Methods Appl. Mech. Eng. Accepted for publication.

[20] A. Huerta, S. Fernández-Méndez, Coupling element free Galerkin and finite element methods, in: Proceedings of the European Congress on Computational Methods in Applied Sciences and Engineering (ECCOMAS 2000), 11-14 September, Barcelona, 2000, electronic publication ISBN: 84-89925-70-4.

[21] S. Fernández-Méndez, A. Huerta, Coupling finite elements and particles for adaptivity: An application to consistently stabilized convection-diffusion, in: M. Griebel, M. A. Schweitzer (Eds.), Meshfree methods for partial differential equations, Vol. 26 of Lecture Notes in Computational Science and Engineering, Springer-Verlag, Berlin, 2002, pp. 117-129, papers from the International workshop, Universität Bonn, Germany, September 11-14, 2001.

[22] A. Huerta, S. Fernández-Méndez, P. Díez, Enrichissement des interpolations d' éléments finis en utilisant des méthodes de particules, ESAIM-Math. Model. Numer. Anal. 36 (6) (2002) 1027-1042.

[23] S. Fernández-Méndez, P. Díez, A. Huerta, Convergence of finite elements enriched with meshless methods, Numer. Math. DOI 10.1007/s00211-003-0465$\mathrm{x}$.

[24] I. Babuska, The finite element method with penalty, Math. Comp. 27 (1973) 221-228.

[25] D. N. Arnold, F. Brezzi, B. Cockburn, L. D. Marini, Unified analysis of discontinuous Galerkin methods for elliptic problems, SIAM J. Numer. Anal. 39 (5) (2001/02) 1749-1779.

[26] J. Nitsche, über ein variations zur lösung von dirichlet-problemen bei verwendung von teilräumen die keinen randbedingungen unterworfen sind, Abh. Math. Se. Univ. 36 (1970) 9-15. 
[27] R. Becker, Mesh adaptation for dirichlet flow control via nitsche's method, Commun. Numer. Methods Eng. 18 (9) (2002) 669-680.

[28] J. Freud, R. Stenberg, On weakly imposed boundary conditions for second order problems, in: Proceeding of the International Conference on Finite Elements in Fluids - New trends and applications, Venezia, 1995.

[29] P. Hansbo, M. G. Larson, Discontinuous Galerkin methods for incompressible and nearly incompressible elasticity by Nitsche's method, Comput. Methods Appl. Mech. Engrg. 191 (17-18) (2002) 1895-1908. 